\title{
Impact of Unreplaced Lost Teeth on Blood Pressure: An Evaluation of Health Checkup Data
}

Keywords: Cardiovascular disease; Dyslipidemia; Epidemiology; Hyperglycemia; Hypertension; Tooth loss

\begin{abstract}
Although positive relationships between tooth loss and cardiovascular disease have been extensively reported, the impact of tooth loss left unreplaced for a certain period on cardiometabolic conditions remains unclear. Thus, we a imed to evaluate the effects of unreplaced tooth loss with cardiometabolic markers in middle-aged bankers using data obtained from health checkups conducted at 2 age points with a 4 -year interval. A total of 218 bankers aged 46 years with unreplaced teeth after loss 4 years prior (at age of 42 years) were analyzed. The health checkup included a physical examination, collection of blood specimen for laboratory test, a self-administered questionnaire regarding health behaviors, and a dental examination. Participants were categorized into 2 groups; those who had unreplaced tooth loss(UTL) and those who did not. Differencesin mean valuesfor systolic and diastolic blood pressure, high-density lipoprotein cholesterol and triglycerides, fasting blood glucose, and $\mathrm{HbAlc}$ between the groups were evaluated using analysis of covariance. A logistic regression model was constructed to calculate the odds ratio of the relation of UTL with high blood pressure (BP) (systolic BP $\geq 130$ $\mathrm{mmHg}$ and/or diastolic $\mathrm{BP} \geq 85 \mathrm{mmHg}$ ) including covariates. We found that higher levels of both systolic and diastolic BP were observed in participants with UTL than in those without (123.4 vs. $119.4 \mathrm{mmHg}$ and 75.9 vs. $71.9 \mathrm{mmHg}$, respectively), with a statistical significance in mean value for diastolic BP $(P<0.01)$. In addition, participants with UTL had greater odds for high blood pressure, after adjusting for the covariates (adjusted odds ratio $=2.58$ [1.25-5.35]). While, there were no significant differences in regard to the mean values for high-density lipoprotein cholesterol, triglycerides, fasting blood glucose, and $\mathrm{HbAlc}$ between the groups. Our results suggest that tooth loss left unreplaced for a certain period is related to increased BP levels in this early middleaged population.
\end{abstract}

\section{Introduction}

Tooth loss is the final consequence of periodontal disease and dental caries. Numerous epidemiological studies have demonstrated a positive relationship between tooth loss and cardiovascular disease [1-4], as well as various cardio-metabolic risk factors such as obesity [5], hypertension [6], and metabolic syndrome [7]. Chewing difficulties and oral function imbalances caused by lost teeth lead to alterations in food selection and consumption [8-10], and the digestive process [11], which have been proposed to be related to the link between tooth loss and cardiovascular disease $[12,13]$. On the other hand, lost teeth can be replaced with removable or fixed dental prostheses, helping to improve oral function [14].

Although a recent study showed that the number of unreplaced teeth was associated with increased risk for cardiovascular mortality [15], the impact of tooth loss left unreplaced over time

\section{Journal of} Oral Biology

\author{
Miki Ojima ${ }^{1 *}$, Atsuo Amano ${ }^{1}$ and Shu Kurata ${ }^{2}$ \\ ${ }^{1}$ Department of Preventive Dentistry, Osaka University Graduate \\ School of Dentistry, Suita, Japan \\ ${ }^{2}$ Dental Clinic, Osaka Health Support Center, Sumitomo Mitsui \\ Banking Corporation, Osaka, Japan \\ *Address for Correspondence \\ Miki Ojima, Assistant Professor, Department of Preventive Dentistry, \\ Osaka University Graduate School of Dentistry, 1-8 Yamadaoka, Suita, \\ Osaka 565-0871, Japan, Tel: +81-6-6879-2922; Fax: +81-6-6879-2925; \\ E-mail: ojima@dent.osaka-u.ac.jp \\ Submission: 19 September 2015 \\ Accepted: 19 October 2015 \\ Published: 20 October 2015 \\ Copyright: (c) 2015 Ojima M, et al. This is an open access article \\ distributed under the Creative Commons Attribution License, which \\ permits unrestricted use, distribution, and reproduction in any medium, \\ provided the original work is properly cited.
}

on cardiometabolic conditions was not examined. Thus, we hypothesized that individuals with unreplaced tooth loss (UTL) have worse cardiometabolic conditions such as high blood pressure (BP), glucose, and lipid metabolism disorder as compared to those without, and that a certain period of time is needed before the effect of UTL on cardio-metabolic conditions appears in clinical findings.

To test our hypothesis, a long-term randomized clinical trial in which individuals with lost teeth are randomly assigned to a "replacement treatment" group or a "no treatment" group would be ideal. However, such studies are difficult to conduct based on ethical perspective. Thus, we obtained data from health checkups conducted at 2 age points with a 4-year interval, and evaluated the effects of UTL on cardiometabolic markers in middle-aged bankers.

\section{Materials and Methods}

The present study was a continuation of our previous work [16], which was performed using secondary data obtained from health checkups conducted at a large bank company in Japan. Although the Industrial Safety and Health Law of Japan require employers to provide annual medical checkups to employees, dental checkups are not required by the law. Bankers of the present company were eligible to receive dental checkups at the ages of 42 and 46 years. All participant annually had regular checkup and showed negligible cardiovascular defect symptoms. Figure 1 illustrates the flow of participants in the study. The study population consisted of bankers who participated in health checkup examinations from January 2006 to December 2011. Of the 3554 participants who underwent dental checkup at the age of 46 years, we analyzed data from 278 who had tooth loss 4 years prior (at the age of 42 years) that was unreplaced. Data for 60 were excluded because of a medical history that included hypertension, dyslipidemia, or hyperglycemia. Thus, findings for 218 participants were used for analysis. This study was approved by the Ethics Committee of Osaka University, Graduate School of Dentistry (H24-E18), and the requirement of written informed consent from each participant was waived by the ethics committee.

The health checkups were conducted at health support centers of 
Citation: Ojima M, Amano A, Kurata S. Impact of Unreplaced Lost Teeth on Blood Pressure: An Evaluation of Health Checkup Data. J Oral Bio. 2015;2(2): 5.

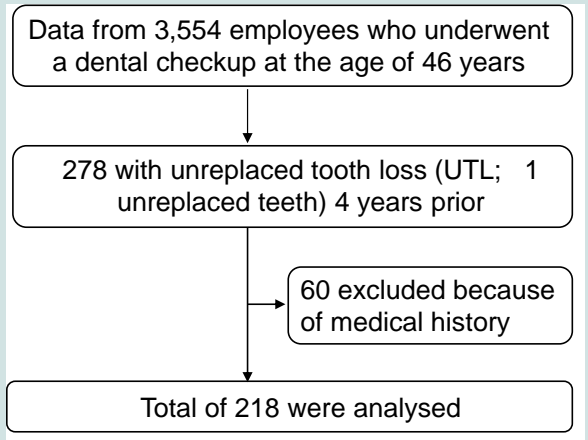

Figure 1: Flowchart of study participants.

the company branches in Osaka and Tokyo. Each medical checkup included a physical examination, collection of blood specimen for laboratory test, and a self-administered questionnaire on health behaviors. The physical examination included measurements of height and weight, and blood pressure (BP). BP was measured by trained nurses using a calibrated automatic electronic BP monitor (HM-701, Elquest Corporation, Chiba, Japan) with participants in a sitting position, after resting for at least 5 minutes. A minimum of 2 measurements were taken on an outstretched arm with a 1to 2-minute interval in each participants. If the reading was high (systolic $\mathrm{BP} \geq 130 \mathrm{mmHg}$ or diastolic $\mathrm{BP} \geq 85 \mathrm{mmHg}$ ), then additional readings were obtained, with the lowest recorded. Routine chemical methods were used to determine fasting blood glucose, high-density lipoprotein cholesterol, and triglycerides. Laboratory analysis details have been described elsewhere [16].

The self-administered questionnaire included questions about alcohol drinking habit, smoking status, and dietary pattern. The dietary section consisted of 4 questions, i.e., "How often do you eat salty foods?" to determine the intake frequency of salty, sweet, and fatty foods, and vegetables. Each answer had 3 choices; often, sometimes, and seldom.

Dental checkups were performed by 8 dentists who were appropriately trained and calibrated according to standardized procedures recommended in the manual published by the World Health Organization [17]. Dental status was recorded on an assessment form developed with reference to the manual. UTL was defined as follows: lost teeth not replaced with a denture, bridge, or implant along with functional problems, presence of prosthesis with functional problems such as fracture, lack of adaptation, and poor retention, or teeth requiring extraction for a prosthesis placement. Third molars were excluded from statistical analysis in this study.

Based on the dental checkup data, the participants were categorized into 2 groups; those who had UTL and those who did not. Body mass index (BMI) was calculated as weight in kilograms divided by the square of the height in meters. Participants were divided based on BMI $\geq 25.0$ and $<25.0$. Alcohol drinking habit was divided into non- and current drinker, while smoking status was classified as never, former, and current. Food intake frequency was classified as often and seldom/sometimes according to answers in the questionnaire.

The characteristics of the participants were compared between

groups using a chi-square test. Differences in mean values for systolic and diastolic BP, fasting blood glucose, high-density lipoprotein cholesterol, and triglycerides were evaluated using analysis of covariance, with gender, BMI, drinking habit, and smoking status as covariates. High blood pressure was defined as systolic $\mathrm{BP} \geq 130$ $\mathrm{mmHg}$ and/or diastolic $\mathrm{BP} \geq 85 \mathrm{mmHg}$. A logistic regression model was constructed to calculate the odds ratio (OR) of the relationship of UTL to hypertension after adjustments for gender, BMI, drinking habit, and smoking status. Statistical analyses were employed using the PASW Statistics 18 statistical software package (IBM Corp., New York, NY, USA). The significance level was set at $\mathrm{P}<0.05$.

\section{Results}

Of the 218 participants who had tooth loss 4 years prior that was unreplaced, 123 (56.4\%) had UTL and 95 (43.6\%) did not at the age of 46 years. The mean number of UTL among those with UTL was 2.2 (standard deviation, 1.6). There were no differences in all study measures between the groups 4 years prior. Table 1 lists the characteristics of participants according to the presence of UTL. The percentage of participants with UTL was significantly higher in men than women. The distributions of drinking habit, smoking status, BMI were comparable in those with and without UTL. In addition, the difference of distribution in food frequency intake between participants with and without UTL was not significant, though the percentage of those who answered that they often eat salty foods was considerably higher in those with UTL than without $(67.3 \%$ vs. $32.7 \%)$.

Figure 2 shows comparisons of adjusted means and 95\% confidence intervals for BP, serum glucose, and lipids. Higher levels of both systolic and diastolic BP were observed in participants with UTL than in those without (123.4 vs. $119.4 \mathrm{mmHg}$, and 75.9 vs. $71.9 \mathrm{mmHg}$, respectively), with a statistical significance in mean

Table 1: Characteristics of participants at the age of 46 years.

\begin{tabular}{|c|c|c|c|}
\hline Characteristics & $\begin{array}{l}\text { Without UTL } \\
(n=95)\end{array}$ & $\begin{array}{l}\text { With UTL } \\
(n=123)\end{array}$ & P-value * \\
\hline \multicolumn{4}{|l|}{ Gender } \\
\hline Male & $81(41.1)$ & $116(58.9)$ & \multirow{2}{*}{0.03} \\
\hline Female & $14(66.7)$ & 7 (33.3) & \\
\hline \multicolumn{4}{|c|}{ Body mass index ( $\mathrm{n}$ and \%) } \\
\hline$<25.0$ & $71(46.1)$ & $83(53.9)$ & \multirow{2}{*}{0.24} \\
\hline$\geq 25.0$ & $24(37.5)$ & $40(62.5)$ & \\
\hline \multicolumn{4}{|c|}{ Drinking habit (n and \%) } \\
\hline Non-drinker & $21(55.3)$ & $17(44.7)$ & \multirow{2}{*}{0.11} \\
\hline Current drinker & $74(41.1)$ & $106(58.9)$ & \\
\hline \multicolumn{4}{|c|}{ Smoking status (n and \%) } \\
\hline Never & $36(46.8)$ & $41(53.2)$ & \multirow{3}{*}{0.78} \\
\hline Former & $17(42.5)$ & $23(57.5)$ & \\
\hline Current & $42(41.6)$ & $59(58.4)$ & \\
\hline \multicolumn{4}{|c|}{ Food intake frequency ( $\mathrm{n}$ and \%) $\dagger$} \\
\hline Salty foods & $16(32.7)$ & $33(67.3)$ & 0.08 \\
\hline Sweet foods & $12(48.0)$ & $13(52.0)$ & 0.64 \\
\hline Fatty foods & $16(41.0)$ & $23(59.0)$ & 0.72 \\
\hline Vegetables & $13(39.4)$ & $20(60.6)$ & 0.60 \\
\hline
\end{tabular}

UTL: Unreplaced Tooth Loss

${ }^{*}$ Chi-square test.

†Number and percentage of participants who answered that they often eat that food. 
Citation: Ojima M, Amano A, Kurata S. Impact of Unreplaced Lost Teeth on Blood Pressure: An Evaluation of Health Checkup Data. J Oral Bio. 2015;2(2): 5.

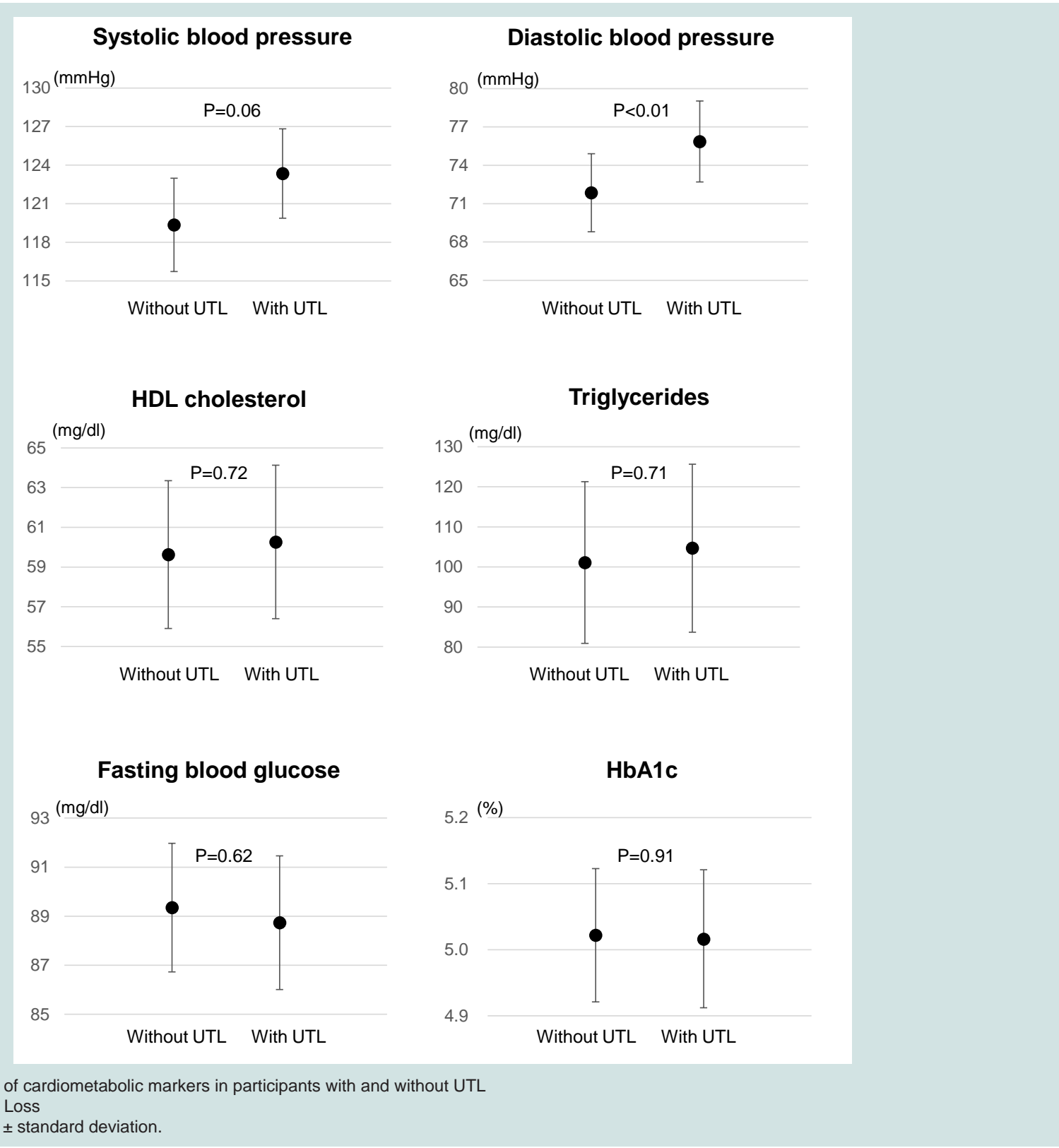

value for diastolic $\mathrm{BP}(\mathrm{P}<0.01)$, while the levels of $\mathrm{HDL}$ cholesterol, triglycerides, fasting blood glucose, and $\mathrm{HbAlc}$ were comparable between them.

Participants with UTL were more likely to have high BP (systolic $\mathrm{BP} \geq 130 \mathrm{mmHg}$ and/or diastolic $\mathrm{BP} \geq 85 \mathrm{mmHg}$ ) as compared to those without UTL ( $37.4 \%$ vs. $17.9 \%$ ). Table 2 shows crude and adjusted ORs for the various study variables in relation to high BP. Following adjustments for gender, BMI, drinking habit, and smoking status, the adjusted OR for high BP in participants with UTL was 2.61 (95\% confidence interval, 1.33 to $5.09, \mathrm{P}<0.01$ ).

\section{Discussion}

In the present study, we evaluated the effects of UTL on cardiometabolic markers, including BP, serum glucose, and lipids, in 46-year-old employees who were found to have tooth loss 4 years prior using health checkup data. Participants with UTL had greater odds (adjusted OR $=2.6$ ) of high BP than those without, which suggests that tooth loss left unreplaced for a certain period is related to increased BP levels in this early middle-aged population. Furthermore, it is interesting to note that a significant difference was only seen for diastolic BP. The Framingham Heart Study reported that in individuals $<50$ years of age, diastolic BP was a more powerful predictor of coronary heart disease risk than systolic BP [18].

The mechanisms related to BP elevation due to UTL may include chronic stress response [19], which is associated with chewing difficulties. Most forms of hypertension are associated with a wide variety of functional changes in the hypothalamus and stress stimulates the release of corticotropin-releasing factor (CRF) in that part of the brain [20], which has been shown to stimulate sympathetic activity, leading to BP elevation [21]. Chewing difficulties and imbalances 
Citation: Ojima M, Amano A, Kurata S. Impact of Unreplaced Lost Teeth on Blood Pressure: An Evaluation of Health Checkup Data. J Oral Bio. 2015;2(2): 5.

ISSN: 2377-987X

Table 2: Odds ratio for having high blood pressure*.

\begin{tabular}{|c|c|c|}
\hline Study variables & Crude OR $(95 \% \mathrm{Cl})$ & Adjusted† OR $(95 \% \mathrm{Cl})$ \\
\hline \multicolumn{3}{|c|}{ Unreplaced tooth loss } \\
\hline Without & 1.00 (Reference) & 1.00 (Reference) \\
\hline With & $2.74(1.45-5.19)$ & 2.61 (1.33-5.09) \\
\hline \multicolumn{3}{|l|}{ Gender } \\
\hline Male & 1.00 (Reference) & 1.00 (Reference) \\
\hline Female & $0.55(0.18-1.71)$ & $0.85(0.23-3.10)$ \\
\hline \multicolumn{3}{|l|}{ Drinking habit } \\
\hline Non-drinker & 1.00 (Reference) & 1.00 (Reference) \\
\hline Current drinker & 3.14 (1.17-8.45) & $2.62(0.91-7.54)$ \\
\hline \multicolumn{3}{|l|}{ Smoking status } \\
\hline Never & 1.00 (Reference) & 1.00 (Reference) \\
\hline Former & $1.57(0.70-3.48)$ & $1.37(0.57-3.30)$ \\
\hline Current & $0.73(0.38-1.43)$ & $0.66(0.32-1.39)$ \\
\hline \multicolumn{3}{|l|}{ Body mass index } \\
\hline$<25.0$ & 1.00 (Reference) & 1.00 (Reference) \\
\hline$\geq 25.0$ & $2.64(1.42-4.92)$ & 2.50 (1.31-4.77) \\
\hline
\end{tabular}

OR: Odds Ratio; $\mathrm{Cl}$ : Confidence Interval

*Systolic blood pressure $\geq 130 \mathrm{mmHg}$ and/or diastolic blood pressure $\geq 85$ $\mathrm{mmHg}$.

†Adjusted for gender, body mass index $(<25.0$, $\geq 25.0$ ), drinking habit (non, current), smoking status (never, former, current).

in oral function for a certain period can cause chronic stress [22]. In rats, occlusal disharmony affected plasma corticosterone levels and noradrenaline release in the hypothalamic paraventricular nucleus [23]. Furthermore, a human study demonstrated an inverse relationship between the level of masticatory performance and sympathetic activity [24].

Several factors to be considered that possibly contribute to the relation of UTL to increased BP levels. Obesity, a well-established risk factor for high $\mathrm{BP}$, has been reported to be associated with an increased risk for tooth loss $[25,26]$. However, the distribution of BMI $(<25.0$ or $\geq 25.0)$ was comparable in participants with and without UTL in this study population. Presence of periodontal disease also increases risk for tooth loss. Most cross-sectional studies have demonstrated a significant positive relationship between periodontal disease and high BP [27-29]. Our data showed no difference in periodontal status assessed by community periodontal index between participants with and without UTL, and no difference in the BP levels by periodontal status (data not shown). Assessment of periodontal condition was not mandatory as part of the dental checkup, and the missing data rate of periodontal status was approximately $30 \%$, accordingly periodontal status was not included in our analysis. Although previous reports have shown that tooth loss causes higher intake of sweet and fatty foods, as well as lower intake of vegetables $[9,10]$, the difference of distribution in the intake frequency of those foods between participants with and without UTL was not significant in the present study.

Participants with UTL showed a tendency for higher intake frequency of salty foods than those without UTL, though the difference was not significant. High salt intake accelerates age-related changes in vasculature [30], resulting in a rise in blood pressure. There could be several reasons for the increased intake frequency of salty foods in those participants. First, foods with a salty taste increase salivary flow [31], which can reduce chewing difficulty due to untreated tooth loss. Furthermore, salty foods may compensate for decreased pleasure in eating that accompanies chewing difficulty [32] and taste dissatisfaction [33], because salt stimulates the brain to release dopamine, a neurotransmitter that helps control the reward and pleasure centers in the brain [34]. Additional studies are needed to determine whether UTL substantially increases the intake of salty foods using a validated food frequency questionnaire [35] or objective assessment of dietary exposure [36].

Our study has several possible limitations. First, we were unable to obtain the exact dates of missing tooth replacement, because our analysis was based on data obtained in health check-up examinations. Thus, the period of time after missing teeth replacement could not be accurately determined. Second, blood pressure measurements were performed in a health check-up setting, thus hypertension was assessed based on blood pressure measurements taken at a single visit, while the Japanese Society of Hypertension recommends that diagnosis of hypertension be based on clinical blood pressure measurements conducted on at least 2 different occasions [37]. Third, socioeconomic status (SES), which is closely related to dental care utilization [38], was not considered in this study because all participants were 46-year-old bankers employed by the same company. In addition, the influence of SES on utilization, if any, may be limited, because the universal health insurance system in Japan covers an extensive range of health services including dental prosthetic treatment. Finally, the study population consisted of employees of a large banc company, thus our results cannot be generalized to other populations.

In conclusion, our results suggest that tooth loss left unreplaced for a certain period is related to increased BP levels in this early middleaged population. Dentists are encouraged to develop treatment plans to help patients prevent and restore tooth loss for systemic health as well as dental health. Further studies are warranted to evaluate the effects of UTL on BP by employing validated assessments of dietary intake.

\section{References}

1. Abnet CC, Qiao YL, Dawsey SM, Dong ZW, Taylor PR, et al. (2005) Tooth loss is associated with increased risk of total death and death from upper gastrointestinal cancer, heart disease, and stroke in a Chinese populationbased cohort. Int J Epidemiol 34: 467-474.

2. Watt RG, Tsakos G, de Oliveira C, Hamer M (2012) Tooth loss and cardiovascular disease mortality risk-results from the Scottish Health Survey. PLoS One 7: e30797.

3. Hung HC, Joshipura KJ, Colditz G, Manson JE, Rimm EB, et al. (2004) The association between tooth loss and coronary heart disease in men and women. J Public Health Dent 64: 209-215.

4. Wiener RC, Sambamoorthi U (2014) Cross-sectional association between the number of missing teeth and cardiovascular disease among adults aged 50 or older: BRFSS 2010. Int J Vasc Med 2014: 6.

5. Östberg AL, Bengtsson C, Lissner L, Hakeberg M (2012) Oral health and obesity indicators. BMC Oral Health 12: 50.

6. Peres MA, Tsakos G, Barbato PR, Silva DA, Peres KG (2012) Tooth loss is associated with increased blood pressure in adults--a multidisciplinary population-based study. J Clin Periodontol 39: 824-833. 
Citation: Ojima M, Amano A, Kurata S. Impact of Unreplaced Lost Teeth on Blood Pressure: An Evaluation of Health Checkup Data. J Oral Bio. 2015;2(2): 5.

7. Holmlund A, Hulthe J, Lind L (2007) Tooth loss is related to the presence of metabolic syndrome and inflammation in elderly subjects: a prospective study of the vasculature in Uppsala seniors (PIVUS). Oral Health Prev Dent 5: 125130

8. Nowjack-Raymer RE, Sheiham A (2007) Numbers of natural teeth, diet, and nutritional status in US adults. J Dent Res 86: 1171-1175.

9. Ritchie CS, Joshipura K, Hung HC, Douglass CW (2002) Nutrition as a mediator in the relation between oral and systemic disease: associations between specific measures of adult oral health and nutrition outcomes. Crit Rev Oral Biol Med 13: 291-300.

10. Wakai K, Naito M, Naito T, Kojima M, Nakagaki H, et al. (2010) Tooth loss and intakes of nutrients and foods: a nationwide survey of Japanese dentists. Community Dent Oral Epidemiol 38: 43-49.

11. N'gom PI, Woda A (2002) Influence of impaired mastication on nutrition. J Prosthet Dent 87: 667-673.

12. Joshipura KJ, Douglass CW, Willett WC (1998) Possible explanations for the tooth loss and cardiovascular disease relationship. Ann Periodontol 3: 175 183.

13. Hung HC, Colditz G, Joshipura KJ (2005) The association between tooth loss and the self-reported intake of selected CVD-related nutrients and foods among US women. Community Dent Oral Epidemiol 33: 167-173.

14. van der Bilt A (2011) Assessment of mastication with implications for oral rehabilitation: a review. J Oral Rehabil 38: 754-780.

15. Schwahn C, Polzer I, Haring R, Dörr M, Wallaschofski H, et al. (2013) Missing, unreplaced teeth and risk of all-cause and cardiovascular mortality. Int J Cardiol 167: 1430-1437.

16. Ojima M, Amano A, Kurata S (2015) Relationship between decayed teeth and metabolic syndrome: data from 4716 middle-aged male Japanese employees. J Epidemiol 25: 204-211.

17. World Health Organization (1997) Oral health surveys: basic methods. $\left(4^{\text {th }} \mathrm{edn}\right)$, World Health Organization, Geneva.

18. Franklin SS, Larson MG, Khan SA, Wong ND, Leip EP, et al. (2001) Does the relation of blood pressure to coronary heart disease risk change with aging? The Framingham Heart Study. Circulation 103: 1245-1249.

19. Lucini D, Di Fede G, Parati G, Pagani M (2005) Impact of chronic psychosocia stress on autonomic cardiovascular regulation in otherwise healthy subjects. Hypertension 46: 1201-1206.

20. Shekhar A, Truitt W, Rainnie D, Sajdyk T (2005) Role of stress, corticotrophin releasing factor (CRF) and amygdala plasticity in chronic anxiety. Stress 8 209-219.

21. Hattori T, Hashimoto K, Ota Z (1986) Brain corticotropin releasing factor in the spontaneously hypertensive rat. Hypertension 8: 1027-1031.

22. Sanders AE, Spencer AJ (2005) Childhood circumstances, psychosocial factors and the social impact of adult oral health. Community Dent Oral Epidemiol 33: 370-377.

23. Yoshihara T, Matsumoto Y, Ogura T (2001) Occlusal disharmony affects plasma corticosterone and hypothalamic noradrenaline release in rats. J Dent Res 80: 2089-2092.
24. Takeuchi N, Ekuni D, Tomofuji T, Morita M (2013) Relationship between masticatory performance and heart rate variability: A pilot study. Acta Odontol Scand 71: 807-812

25. Ostberg AL, Nyholm M, Gullberg B, Råstam L, Lindblad U (2009) Tooth loss and obesity in a defined Swedish population. Scand J Public Health 37: 427433.

26. Bernardo Cde O, Boing AF, Vasconcelos Fde A, Peres KG, Peres MA (2012) Association between tooth loss and obesity in Brazilian adults: a population based study. Rev Saude Publica 46: 834-842.

27. Holmlund A, Holm G, Lind L (2006) Severity of periodontal disease and number of remaining teeth are related to the prevalence of myocardial infarction and hypertension in a study based on 4,254 subjects. J Periodontol 77: $1173-1178$

28. Desvarieux M, Demmer RT, Jacobs DR Jr, Rundek T, Boden-Albala B, et al. (2010) Periodontal bacteria and hypertension: the oral infections and vascular disease epidemiology study (INVEST). J Hypertens 28: 1413-1421.

29. Tsioufis C, Kasiakogias A, Thomopoulos C, Stefanadis C (2011) Periodontitis and blood pressure: the concept of dental hypertension. Atherosclerosis 219: $1-9$

30. Avolio AP, Deng FQ, Li WQ, Luo YF, Huang ZD, et al. (1985) Effects of aging on arterial distensibility in populations with high and low prevalence of hypertension: comparison between urban and rural communities in China. Circulation 71: 202-210

31. Dawes C, Watanabe S (1987) The effect of taste adaptation on salivary flow rate and salivary sugar clearance. J Dent Res 66: 740-744.

32. Klein BE, Klein R, Knudtson MD (2004) Life-style correlates of tooth loss in an adult Midwestern population. J Public Health Dent 64: 145-150.

33. Yoshinaka M, Yoshinaka MF, Ikebe K, Shimanuki Y, Nokubi T (2007) Factors associated with taste dissatisfaction in the elderly. J Oral Rehabil 34: 497502.

34. Corsica JA, Pelchat ML (2010) Food addiction: true or false? Curr Opin Gastroenterol 26: 165-169.

35. Ferrara LA, Ricci F, Viola S, DE Luca G, Ferrara F, et al. (2007) Dietary pattern and blood pressure control in a hypertension outpatient clinic. Hypertens Res 30: 1043-1050.

36. Tanaka T, Okamura T, Miura K, Kadowaki T, Ueshima H, et al. (2002) A simple method to estimate populational 24-h urinary sodium and potassium excretion using a casual urine specimen. J Hum Hypertens 16: 97-103.

37. Ogihara T, Kikuchi K, Matsuoka H, Fujita T, Higaki J, et al. (2009) The Japanese Society of Hypertension Guidelines for the Management of Hypertension (JSH 2009). Hypertens Res 32: 3-107.

38. Wamala S, Merlo J, Boström G (2006) Inequity in access to dental care services explains current socioeconomic disparities in oral health: the Swedish National Surveys of Public Health 2004-2005. J Epidemiol Community Health 60: 1027-1033.

\section{Acknowledgements}

This study was supported by a Grant-in-Aid for Scientific Research (C) (No. 24593148) and a Grant-in-Aid for Challenge to Intractable Oral Diseases of Osaka University. The authors thank Dr. Haruhito Tatsuma, Dr. Gentaro Watanabe, Dr. Fumitaka Goto, and Dr. Kyohei Kajiwara, as well as other staff at the Health Support Center, Sumitomo Mitsui Banking Corporation, for their contributions in acquisition of data. 